\title{
Folic Acid Improves the Inflammatory Response in LPS-Activated THP-1 Macrophages
}

\author{
Mirian Samblas $\mathbb{D}^{1,2}$ J. Alfredo Martínez $\mathbb{D}^{1,2,3,4}$ and Fermín Milagro $\mathbb{D}^{1,2}$ \\ ${ }^{1}$ Department of Nutrition, Food Science, and Physiology, Centre for Nutrition Research, University of Navarra, Pamplona, Spain \\ ${ }^{2}$ Centro de Investigación Biomédica en Red de la Fisiopatología de la Obesidad y Nutrición (CIBERobn), Instituto de Salud Carlos III, \\ Madrid, Spain \\ ${ }^{3}$ Instituto de Investigación Sanitaria de Navarra (IdiSNA), Pamplona, Spain \\ ${ }^{4}$ IMDEA Food, Madrid, Spain
}

Correspondence should be addressed to Fermín Milagro; fmilagro@unav.es

Received 16 January 2018; Revised 19 April 2018; Accepted 16 May 2018; Published 4 July 2018

Academic Editor: José C. Rosa

Copyright ( 2018 Mirian Samblas et al. This is an open access article distributed under the Creative Commons Attribution License, which permits unrestricted use, distribution, and reproduction in any medium, provided the original work is properly cited.

\begin{abstract}
DNA methylation has been suggested as a regulatory mechanism behind some inflammatory processes. The physiological actions of methyl donors, such as folic acid, choline, and vitamin $\mathrm{B}_{12}$ on inflammation-related disease have been associated with the synthesis of the universal methyl donor S-adenosyl methionine (SAM). The aim of this study was to evaluate the effects of folic acid, choline, vitamin $\mathrm{B}_{12}$, and a combination of all on preventing the lipopolysaccharide- (LPS-) induced inflammatory response in human THP-1 monocyte/macrophage cells. Folic acid and the mixture of methyl donors reduced interleukin 1 beta (IL1B) and tumour necrosis factor (TNF) expression as well as protein secretion by these cells. Folic acid and choline decreased C-C motif chemokine ligand 2 (CCL2) mRNA levels. In addition to this, the methyl donor mixture reduced Cluster of differentiation 40 (CD40) expression, but increased serpin family E member 1 (SERPINE1) expression. All methyl donors increased methylation levels in CpGs located in IL1B, SERPINE1, and interleukin 18 (IL18) genes. However, TNF methylation was not modified. After treatment with folic acid and the methyl donor mixture, ChIP analysis showed no change in the binding affinity of nuclear factor $-\kappa \mathrm{B}(\mathrm{NF}-\kappa \mathrm{B})$ to $I L 1 B$ and TNF promoter regions after the treatment with folic acid and the methyl donor mixture. The findings of this study suggest that folic acid might contribute to the control of chronic inflammation in inflammatory-related disease.
\end{abstract}

\section{Introduction}

Inflammation is traditionally defined as the short-term adaptive response to fight against injury, caused by pathogens or biological and chemical stimuli [1]. Although acute inflammation is a crucial component for maintaining homeostasis in the body, persistent and chronic inflammation is involved in the development of several clinical manifestations and diseases. During inflammatory disease, monocytes and macrophages produce cytokines in response to different stimuli, such as lipopolysaccharide (LPS) [2]. The proinflammatory molecules released by macrophages in the inflamed regions orchestrate the enhancement of monocyte recruitment from blood to tissue. Recruited monocytes differentiate into macrophages to continue the inflammatory response [3].
Alongside this, studies have described that epigenetic mechanisms contribute to the pathogenesis of several chronic inflammatory-related diseases by regulating important steps such as macrophage infiltration or cytokine secretion $[4,5]$. For instance, inflammatory genes like interleukins (IL) IL6, $I L 4, I L 8, I L 1 B$, or interferon INF- $\gamma$ have been described to be methylated differently in several chronic inflammatory diseases $[4,6]$.

Methionine, folate, betaine, choline, and vitamins $B_{2}, B_{6}$, and $\mathrm{B}_{12}$ are considered methyl donor precursors naturally occurring in the diet [7]. These substances participate in the methionine pathway for the synthesis of S-adenosyl methionine (SAM), which is the universal methyl donor for DNA methylation reactions [8]. The anti-inflammatory effects exerted by some of these compounds in a variety of 
inflammatory diseases have been described in previous research. For example, folic acid supplementation improved disease outcomes in subjects with hypertension, diabetes, and stroke by reducing levels of inflammatory markers (CRP, VCAM-1, IL-1 $\beta$, and TNF- $\alpha$ ) [9]. In addition to this, the combined supplementation of folate and vitamin $B_{12}$ ameliorated inflammation during pregnancy by modifying the concentration of inflammatory cytokines [10]. Lastly, vitamin $B_{12}$ has been negatively associated with proinflammatory cytokines and low-grade systemic inflammation [11]. On the other hand, deficiencies in the abovementioned methyl donors have also been shown to lead to adverse effects $[10,12]$. For example, vitamin $B_{12}$ deficiency has been related to metabolic disturbances such as hyperhomocysteinemia, obesity, hypertension, and insulin resistance [10]. Choline deficiency has been associated with the development of fatty liver disease and demonstrated to worsen the outcome of liver fibrosis in patients with nonalcoholic steatohepatitis (NASH) [12]. Interestingly, NASH patients that were choline-deficient exhibited amelioration of steatohepatitis after choline supplementation [13]. Similarly, methyl donor supplementation prevented HFS diet-induced liver fat accumulation in rats fed an obesogenic diet [14].

The anti-inflammatory effect of methyl donors in monocytes before differentiation and LPS-induced inflammatory response in macrophages, along with the role of DNA methylation in this process, have been scarcely studied. For this reason, the aim of this study was to investigate the effects of methyl donors, both individually and together, on the attenuation of LPS-induced inflammatory response and the possible underlying epigenetic mechanisms in human THP-1 monocyte/macrophage cells. Monocytes were incubated with folic acid, choline, and vitamin $\mathrm{B}_{12}$ or a methyl donor mixture of folic acid, choline, and vitamin $\mathrm{B}_{12}$. Monocytes were then differentiated into macrophages and an inflammatory response was induced with LPS.

\section{Material and Methods}

2.1. Reagents. Folic acid, vitamin $\mathrm{B}_{12}$, and choline chloride were supplied from Sigma-Aldrich (MO, USA). Phorbol 12-myristate 13-acetate (TPA) (Sigma-Aldrich) was used for differentiating THP-1 monocytes into macrophage-like cells. LPS from E. coli K12 strain (Invitrogen, CA, USA) was applied to activate macrophages. Thiazolyl Blue Tetrazolium Bromide (MTT) (Sigma-Aldrich) was used to investigate the toxic effects of methyl donors on THP-1 cells.

2.2. Cell Culture and Treatments. Human monocyte THP-1 cells were purchased from American Type Cell Culture (ATCC $^{\circledR}$ TIB-202 ${ }^{\mathrm{TM}}$, VA, USA). Cells were maintained at $37^{\circ} \mathrm{C}$ and $5 \% \mathrm{CO}_{2}$ in RPMI-1640 medium (Gibco) modified to contain $2 \mathrm{mM}$ L-glutamine, $1 \mathrm{mM}$ sodium pyruvate, $4.5 \mathrm{~g} / \mathrm{l}$ glucose, and $1.5 \mathrm{~g} / \mathrm{l}$ sodium bicarbonate and supplemented with $10 \%$ fetal bovine serum (GIBCO), $100 \mathrm{U} / \mathrm{ml}$ penicillin, and $100 \mu \mathrm{g} / \mathrm{ml}$ streptomycin.

THP-1 cells were treated with $11.3 \mu \mathrm{M}$ folic acid, dissolved in $1 \mathrm{M} \mathrm{NaOH}, 105 \mu \mathrm{M}$ choline chloride, $18.5 \mathrm{nM}$ vitamin $\mathrm{B}_{12}$, and a mixture of methyl donors consisting of folic acid, choline chloride, and vitamin $\mathrm{B}_{12}$ at the concentrations previously indicated. Concentrations were determined by multiplying ten times the basal concentration present in the RPMI-1640 medium for each compound. After 24h, cells were differentiated into macrophages by incubation with $25 \mathrm{ng} / \mathrm{ml}$ TPA for $48 \mathrm{~h}$ and then were activated with $100 \mathrm{ng} / \mathrm{ml}$ LPS for 24 hours. Finally, RNA and DNA were extracted and supernatants were collected for ELISA analysis.

2.3. Cell Viability Analysis. For the viability assay, THP-1 cells were pretreated with methyl donors at the selected concentrations during $24 \mathrm{~h}$, as described above, in a 96-well plate. After the treatments, $20 \mu \mathrm{l} \mathrm{MTT}(5 \mathrm{mg} / \mathrm{ml})$ was added to each well and plates were incubated for $2 \mathrm{~h}$ at $37^{\circ} \mathrm{C}$. Formazan crystal formation was solubilised in $100 \mu \mathrm{l} /$ well DMF-glacial acetic acid-SDS solution consisting of $40 \%$ DMF, $2 \%$ glacial acetic acid, and $16 \% w / v$ sodium SDS. Formazan production was quantified by absorbance at $570 \mathrm{~nm}$ using a microplate reader (Multiskan Spectrum, Thermo Electron Corporation, Finland). The results were expressed as relative cell viability (\%).

2.4. Analysis of $m R N A$ Expression by Quantitative Real-Time $P C R$. Total RNA was extracted from cells with TRizol ${ }^{\circledR}$ Reagent (Invitrogen). RNA quality and concentration were measured using the Nanodrop Spectrophotometer ND1000 (Thermo Fisher Scientific, MA, USA). Approximately $1 \mu \mathrm{g}$ of total RNA was reverse-transcribed into cDNA by the MultiScribe $^{\mathrm{TM}}$ Reverse Transcriptase Kit, following the manufacturer's instructions (Thermo Fisher Scientific, MA, USA). Real-time PCR was performed using ABI Prism 7900HT Sequence Detection System and Taqman Universal Master Mix (Applied Biosystems, CA, USA). Predesigned TaqMan primers and probes for IL1B (Hs01555410_m1), TNF (Hs00174128_m1), IL18 (Hs01038788_m1), SERPINE1 (Hs01126606_m1), CD40 (Hs01002913_g1), CCL2 (Hs00234140_m1), and TLR4 (Hs00152939_m1) genes were used (Applied Biosystems). The levels of these mRNAs were normalized to the level of GAPDH (Hs02758991_g1) mRNA expression. Relative expression was determined by using the comparative $2^{-\Delta \Delta \mathrm{Ct}}$ method.

2.5. Cytokine Secretion Analysis by Enzyme-Linked Immunosorbent Assay (ELISA). Culture supernatants were collected after the treatments and stored at $-80^{\circ} \mathrm{C}$ for further cytokine analysis. Protein concentrations of IL- $1 \beta$, TNF- $\alpha$, PAI1, and CD40 were measured with standard ELISA kits (R\&D Systems Europe, UK), according to the manufacturer's protocols. Absorbance was measured at $450 \mathrm{~nm}$ using a microplate reader (Multiskan Spectrum, Thermo Electron Corporation, Finland).

2.6. DNA Methylation Analysis by MALDI-TOF Mass Spectrometry. DNA was isolated using MasterPure ${ }^{\mathrm{TM}}$ DNA Purification Kit (Illumina, WI, USA), according to the manufacturer's guidelines. Genomic DNA was sodium bisulfite-converted using the EpiTect Bisulfite Kit (Qiagen, CA, USA). DNA methylation quantification was performed by MassARRAY EpiTYPER technology (Sequenom Inc., CA, USA). This method uses matrix-assisted laser desorption 
ionization time-of-flight (MALDI-TOF) mass spectrometry in combination with RNA base-specific cleavage (MassCLEAVE). Four amplicons covering $32 \mathrm{CpG}$ sites were selected. EpiDesigner software (Sequenom Inc; http://www. epidesigner.com/start3.html) was used to design PCR primers for the amplicons of interest: IL1B (chr2: 112,837,566112,837,895), TNF (chr6: 31,575,209-31,575,481), SERPINE1 (chr7: 101,127,068-101,127,411), and IL18 (chr11: $112,163,853-112,164,105)$. The designed primers are shown in Supplementary Table 1 and the complete amplicon sequences are reported in Supplementary Figure 1. The complete methodology was previously explained [15].

2.7. Chromatin Immunoprecipitation (ChIP) Assay. ChiP assay was performed with the ChIP-IT ${ }^{\mathrm{TM}}$ Express Enzymatic Kit (Active Motif, CA, USA), following the manufacturer's guidelines. THP-1 cells were cultured for 24 hours with methyl donors and were then differentiated with TPA $(25 \mathrm{ng} / \mu \mathrm{l})$ for 48 hours and activated with LPS (100 $\mathrm{ng} / \mu \mathrm{l})$ over 24 hours. The cell medium was discarded and 36.5\% formaldehyde was added directly to the cell surface for $10 \mathrm{~min}$. Cross-linking between proteins and DNA was stopped by the addition of glycine for $5 \mathrm{~min}$ at room temperature and cells were collected by scraping. Then, cells were incubated with lysis buffer for $30 \mathrm{~min}$ at $4^{\circ} \mathrm{C}$ and DNA was fragmented via enzyme-based digestion for $10 \mathrm{~min}$ at $37^{\circ} \mathrm{C}$. Chromatin was immunoprecipitated using rabbit polyclonal antibody to nuclear factor $-\kappa \mathrm{B}(\mathrm{NF}-\kappa \mathrm{B}$; ab7970, Abcam, MA, USA). After immunoprecipitation, cross-linking of protein-DNA complexes was reversed. Real-time quantitative PCR was performed using primers for $I L 1 B$ : sense $5^{\prime}$-agcaacaaagctgccactta- $3^{\prime}$ and antisense $5^{\prime}$-tgacgtgctgtgtgaatttg- $3^{\prime}$, and TNF: sense $5^{\prime}$-ggagaatgtccagggctatg- $3^{\prime}$ and antisense $5^{\prime}$-tcctggaggctctttcactc- $3^{\prime}$.

2.8. Transcription Factor-Binding Site Analysis. In order to identify the putative transcription factor binding site in the CpG sites of the ILIB gene, a bioinformatic analysis was performed through LASAGNA-Search 2.0 using TRANSFAC matrices and aligned models, as described elsewhere [16].

2.9. Statistical Analysis. Normality was assessed by Kolmogorov-Smirnov and Shapiro-Wilk tests. For the statistical analysis of the results, a one-way ANOVA followed by Dunnett's test for multiple comparisons between groups and an unpaired Student $t$-test, for the direct comparisons between two groups, were used. Differences were considered significant at $P$ value $<0.05$. Statistics were performed using Prism 5.0 (GraphPad Software, CA, USA).

\section{Results}

3.1. Methyl Donors Did Not Affect Cell Viability. Cell viability was measured by MTT assay after incubation with folic acid at $11.3 \mu \mathrm{M}$, choline at $105 \mu \mathrm{M}$, vitamin $\mathrm{B}_{12}$ at $18.5 \mathrm{nM}$, and the methyl donor mixture. The selected concentrations were within the range proposed by previous studies $[9,17,18]$. Cell viability was not significantly affected by methyl donors at these concentrations (Supplementary Figure 2).
3.2. Effects of Methyl Donors on the Expression of Genes Associated with the Inflammatory Response in THP-1 Macrophages Activated with LPS. Treatment of THP-1 cells with the different compounds before the differentiation with TPA and activation with LPS altered the expression of most of the inflammation-related genes compared to the control treatment (Figure 1). Folic acid and the methyl donor mixture reduced $I L 1 B(P<0.05$ for folic acid; $P<0.01$ for the methyl donor mixture) and TNF ( $P<0.05$ for folic acid; $P<0.001$ for methyl donor mixture) mRNA expression. Folic acid also reduced TLR4 $(P<0.05)$ and CCL2 $(P<0.05)$, but increased SERPINE1 $(P<0.05)$ gene expression. Moreover, methyl donor mixture incubation reduced the levels of CD40 $(P<0.05)$ but increased SERPINE1 $(P<0.05)$. Choline decreased the expression of CCL2 $(P<0.05)$. However, no statistically significant changes were observed after vitamin $\mathrm{B}_{12}$ incubation.

3.3. The Pretreatment with Folic Acid and Methyl Donor Mixture Reduced IL-1 $\beta$ and TNF- $\alpha$ Secretion of LPSActivated Macrophages. Concerning cytokine secretion, the incubation with folic acid and the methyl donor mixture reduced the levels of IL-1 $\beta(P<0.01)$ and TNF- $\alpha(P<0.01$ and $P<0.05$, resp.), but not CD40 and PAI-1. However, no changes were observed with the other methyl donors (Figure 2).

3.4. Incubation with Methyl Donors Increased DNA Methylation in the Inflammatory Genes. The regions studied in the genes ILIB, SERPINE1, and IL18 displayed an overall gain of methylation when LPS-activated macrophages were treated with the different methyl donors. This hypermethylation was especially significant after the incubation with folic acid. As shown in Table 1, folic acid significantly increased $(P<0.05)$ the methylation levels of $\mathrm{CpG}_{-} 1(190 \%), \mathrm{CpG} \_5$ (680\%), and CpG_6 (200\%) of IL1B, CpG_1 (750\%), CpG_2 (88\%), CpG_3.4 (136\%), CpG_7 (1003\%), and CpG_9 (88\%) of SERPINE1, and CpG_4 (53\%) and CpG_5 (27\%) of IL18 when compared with the methylation percentage of the nontreated LPS-activated macrophages. No changes in methylation were noted in the analyzed region of TNF after the treatment. Regarding choline chloride, vita$\min \mathrm{B}_{12}$, and the methyl donor mixture, incubation with these compounds also significantly increased $(P<0.05)$ the methylation levels of some CpG sites concerning the studied genes (Table 1).

3.5. NF- $\kappa B$ Binding to IL-1 $\beta$ and TNF- $\alpha$ Was Not Affected after the Incubation with Folic Acid and the Methyl Donor Mixture. IL1B and TNF gene expression and secretion decreased after the incubation with folic acid and the methyl donor mixture (Figures 1 and 2). IL1B DNA methylation levels increased; TNF methylation levels did not. To determine the effect of DNA methylation in the sequence of proinflammatory genes on NF- $\kappa \mathrm{B}$ binding to $I L 1 B$ and TNF promoters, a ChIP assay was performed. The analysis showed no significant changes in NF- $\kappa \mathrm{B}$ binding to $I L 1 B$ and TNF promoter regions in THP-1 cells treated with folic acid and the methyl donor mixture (Figure 3). 

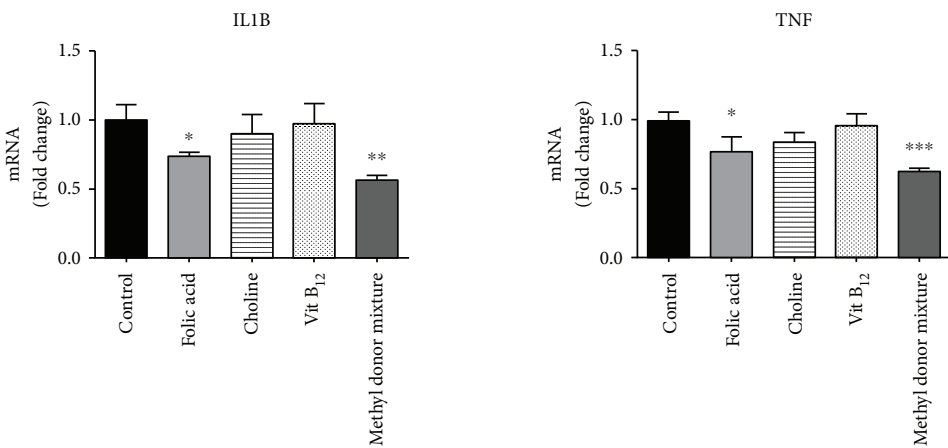

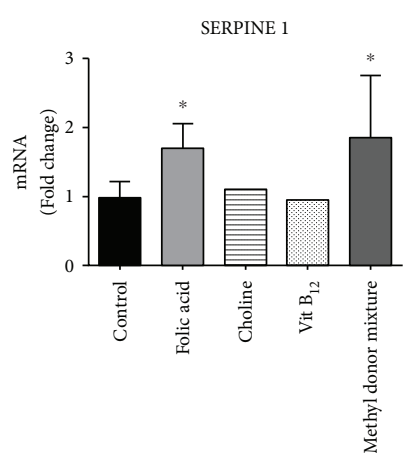

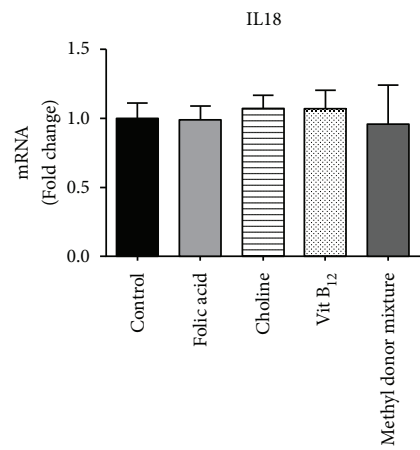
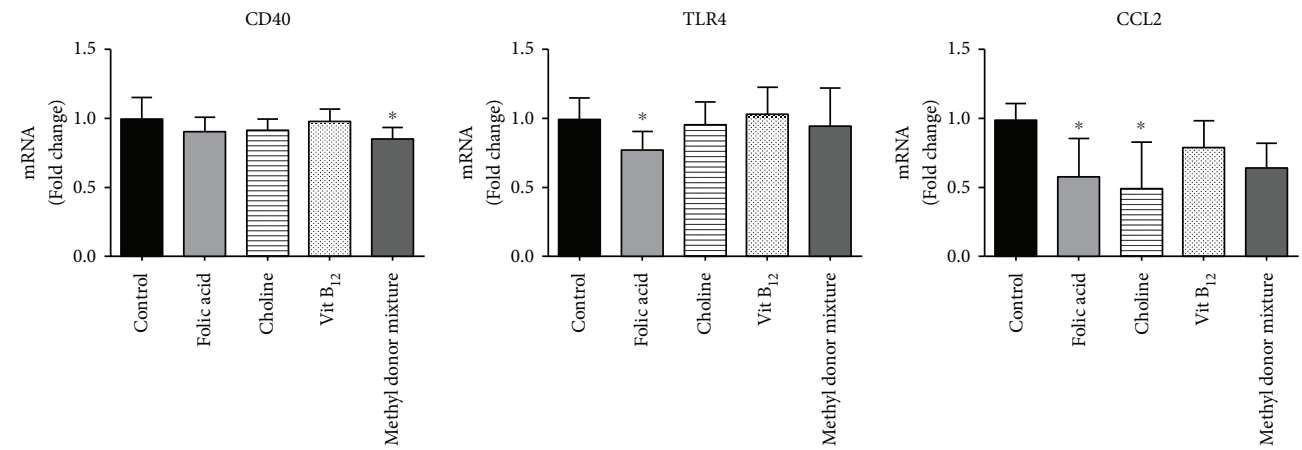

FIGURE 1: Effects of folic acid, choline, vitamin $\mathrm{B}_{12}$, and the methyl donor mixture on the expression of inflammatory genes in THP-1 cells treated with TPA and LPS. Results are expressed as means \pm SD $(n=8)$. Differences between groups were analyzed by one-way ANOVA followed by Dunnett's test. ${ }^{*} P$ value $<0.05,{ }^{* *} P$ value $<0.01$, and ${ }^{* * *} P$ value $<0.001$ versus control.

\section{Discussion}

Previous studies in humans have analyzed the association between folic acid and inflammation. For example, a casecontrol study showed a reduction of cytokine levels after a 12-week treatment with folic acid [19]. In addition, folic acid supplementation in patients with a high risk of coronary artery disease was associated with a reduction in proinflammatory cytokines (e.g., monocyte chemoattractant protein 1 or MCP-1) in human monocytes [20]. In the present study, we demonstrated that folic acid and a mixture of methyl donors reduced the expression of proinflammatory genes (e.g., TNF, IL1B, CD40, CCL2, and TLR4) in THP-1 monocytes, when the monocytes were differentiated into macrophages and activated with LPS. In agreement with our results, the incubation of murine monocyte RAW 264.7 cells with folic acid reduced the expression of proinflammatory genes during LPS activation [17]. In contrast, folic acid deficiency in the same cell line enhanced the expression of proinflammatory genes [21].

Current data revealed that folic acid and the methyl donor mixture not only reduce proinflammatory gene expression in THP-1 monocytes, but also decrease the secretion of TNF- $\alpha$ and IL- $1 \beta$ cytokines when cells were differentiated to macrophages and activated by LPS.

During the inflammatory response, the proinflammatory mediators, specially MCP-1 (encoded by the CCL2 gene), contribute to the migration of circulatory monocytes into the surrounding tissue [22], by specifically attracting monocytes towards the inflamed area, promoting tissue damage and disease. In this context, our results suggest that folic acid and a mixture of methyl donors could reduce the inflammatory response of the monocytes and the macrophages derived from these monocytes would decrease cytokine and chemokine secretion.

The specific mechanisms for the beneficial effects of folic acid or methyl donors on inflammation have not been clearly elucidated. One of the possible explanations is epigenetics, via DNA and histone methylation [23]. Folate, choline, and vitamin $B_{12}$ directly participate in the formation of S-adenosyl methionine (SAM), which is the major donor of methyl groups for DNA methylation [24]. In the current trial, folic acid and the methyl donor mixture increased methylation levels of IL1B, SERPINE1, and IL18. However, only $I L 1 B$ presented lower gene expression and protein secretion, which was associated with hypermethylation after folic acid supplementation. This result suggests that $I L 1 B$ gene expression may be modulated by changes in DNA methylation induced by folic acid. However, the methylation changes of SERPINE1 and IL18 did not correlate with changes in gene expression. Incubation with methyl donors was for 24 hours and then the monocytes were differentiated for 48 hours and activated by LPS for a further 24 hours. A recent study of $I L 18$ expression in LPS-stimulated murine macrophages showed that the maximum level of expression of this interleukin was 3-6 hours after induction. No changes were found after 24 hours suggesting an earlier enzymatic activation of poly(ADP-ribose) 


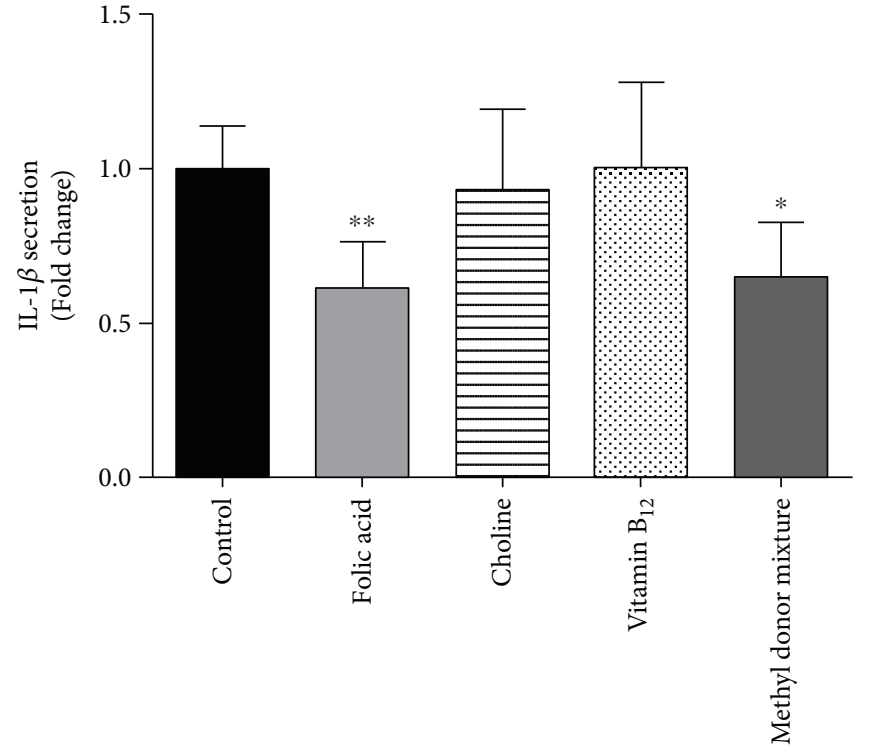

(a)

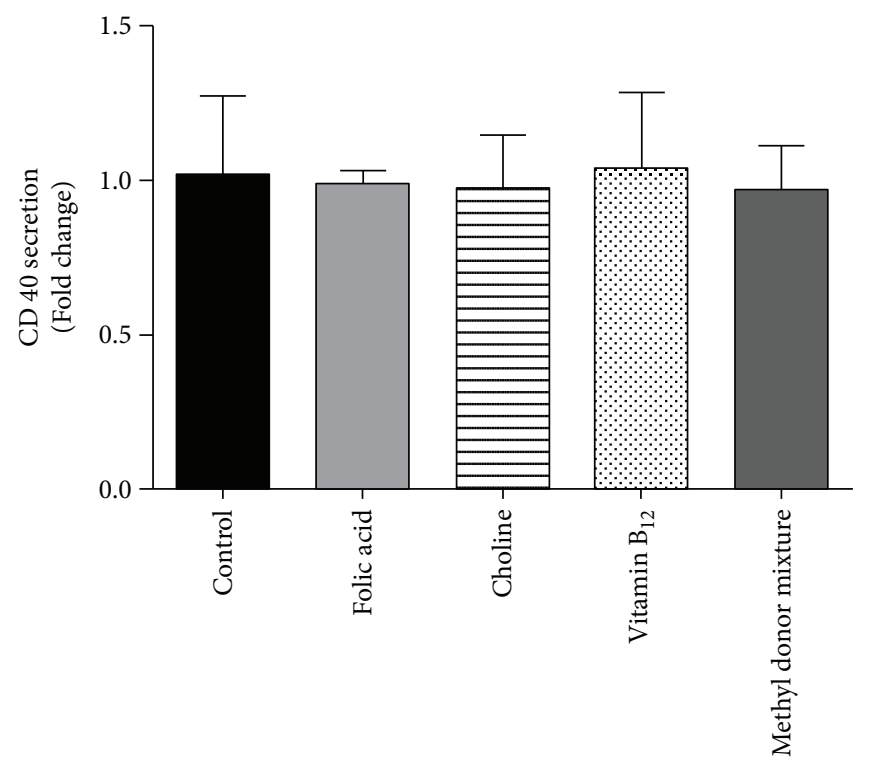

(c)

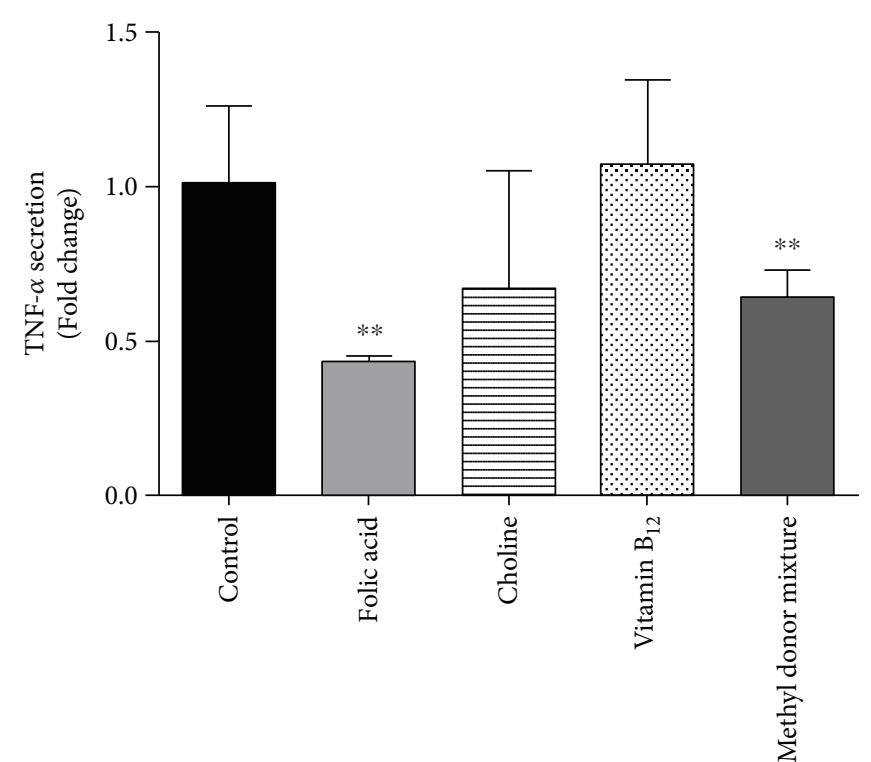

(b)

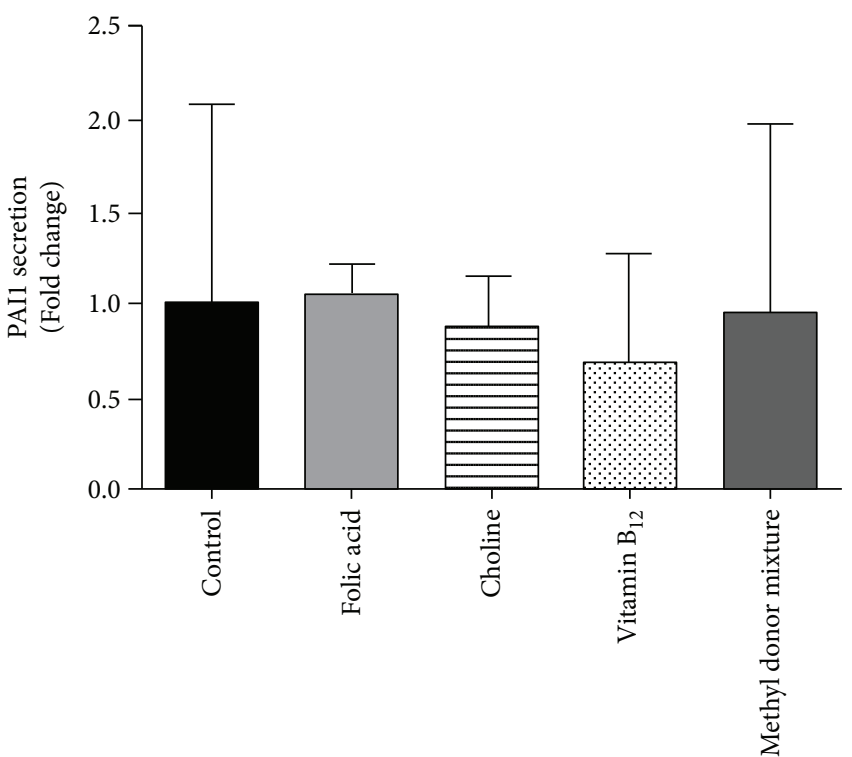

(d)

FIGURE 2: Effects of folic acid, choline, vitamin $\mathrm{B}_{12}$, and the methyl donor mixture on the secretion of inflammatory cytokines in THP-1 cells treated with TPA and LPS. (a) IL- $1 \beta$, (b) TNF- $\alpha$, (c) CD40, and (d) PAI-1 secretion. Results are expressed as means \pm SD ( $n=7-8)$. Differences between groups were analyzed by one-way ANOVA followed by Dunnett's test. ${ }^{*} P$ value $<0.05$ and ${ }^{* *} P$ value $<0.01$.

polymerase 1 (PARP-1) that induces IL18 expression [24]. In this context, the time or the concentration of supplements might have been insufficient to evidence subtle changes in IL18 and SERPINE1 expression.

Unexpectedly, no changes in DNA methylation levels in the TNF gene were found after the treatment with methyl donors. In line with these results, Kolb and Petrie [21] found that although folate deficiency in murine macrophages reduced DNA methyltransferase expression, TNF DNA methylation did not change. In addition, in murine macrophages incubation with exogenous SAM attenuated the LPS-stimulated expression of TNF [25]. The reduction in
TNF expression without a change in methylation could be due to folic acid causing upstream methylation. Recent studies have uncovered a new epigenetic mechanism for gene expression regulation: mRNA methylation. In vitro data has shown that methyladenine of mRNA influences mRNA transcription, splicing, nuclear export, translation, and mRNA stability $[26,27]$. Therefore, RNA methylation may also be a regulatory mechanism altering TNF expression after the supplementation with the methyl donors.

Transcriptional activation of TNF and IL1B by LPS requires the stimulation of a set of pathways and transcription factors, including NF- $\kappa \mathrm{B}$, early growth response protein 
TABLE 1: CpG methylation levels (as percentage) in IL1B, TNF, IL18, and SERPINE1 genes after the incubation of THP-1 monocytes with folic acid, choline, vitamin $\mathrm{B}_{12}$, and the methyl donor mixture, measured by MassARRAY. Unpaired Student's $t$-test was used to compare each CpG with the control group. ${ }^{*} P$ value $<0.05 ;{ }^{* *} P$ value $<0.01$; and ${ }^{* * *} P$ value $<0.001$.

\begin{tabular}{|c|c|c|c|c|c|}
\hline & Control & Folic acid & Choline & Vitamin $B_{12}$ & Methyl donor mixture \\
\hline \multicolumn{6}{|l|}{$\overline{I L 1 B}$} \\
\hline CpG_1 & $6.88 \pm 3.97$ & $15.5 \pm 6.14^{*}$ & $11.4 \pm 1.55^{*}$ & $9.38 \pm 0.95$ & $14.0 \pm 3.11^{*}$ \\
\hline CpG_2 & $94.9 \pm 3.14$ & $96.2 \pm 1.66$ & $94.8 \pm 2.06$ & $9.76 \pm 0.85$ & $96.9 \pm 0.85$ \\
\hline CpG_3 & $2.88 \pm 2.09$ & $1.75 \pm 1.26$ & $5.75 \pm 1.77$ & $3.75 \pm 3.89$ & $3.12 \pm 3.75$ \\
\hline CpG_4 & $2.75 \pm 2.06$ & $1.88 \pm 1.79$ & $1.25 \pm 1.19$ & $1.75 \pm 0.96$ & $3.75 \pm 1.55$ \\
\hline CpG_5 & $2.12 \pm 2.49$ & $6.50 \pm 4.06^{*}$ & $1.25 \pm 0.64$ & $3.25 \pm 1.55$ & $10.0 \pm 1.13$ \\
\hline CpG_6 & $0.75 \pm 0.50$ & $7.12 \pm 6.14^{*}$ & $9.50 \pm 0.58^{* * *}$ & $9.25 \pm 2.33^{* * *}$ & $0.88 \pm 0.48$ \\
\hline \multicolumn{6}{|l|}{$T N F$} \\
\hline CpG_1 & $98.2 \pm 1.32$ & $96.1 \pm 2.78$ & $98.0 \pm 1.47$ & $95.2 \pm 2.72$ & $95.8 \pm 3.07$ \\
\hline CpG_2 & $67.8 \pm 3.95$ & $64.8 \pm 7.59$ & $65.8 \pm 4.48$ & $69.9 \pm 1.93$ & $63.4 \pm 6.46$ \\
\hline CpG_3 & $48.1 \pm 12.6$ & $48.6 \pm 8.53$ & $51.2 \pm 10.3$ & $47.9 \pm 4.71$ & $45.0 \pm 4.65$ \\
\hline CpG_4.5.6 & $19.9 \pm 6.76$ & $15.2 \pm 4.41$ & $17.4 \pm 3.09$ & $19.8 \pm 0.87$ & $17.8 \pm 1.79$ \\
\hline CpG_8 & $33.9 \pm 3.49$ & $29.6 \pm 4.37$ & $31.8 \pm 3.93$ & $34.2 \pm 2.53$ & $34.0 \pm 3.24$ \\
\hline \multicolumn{6}{|l|}{ IL18 } \\
\hline CpG_1 & $12.5 \pm 17.4$ & $2.0 \pm 0.5$ & $2.12 \pm 0.85^{*}$ & $1.25 \pm 1.32$ & $2.5 \pm 2.0$ \\
\hline $\mathrm{CpG \_ 2}$ & $6.88 \pm 1.55$ & $6.0 \pm 1.22$ & $7.38 \pm 2.06^{*}$ & $9.50 \pm 1.0$ & $10.1 \pm 2.25$ \\
\hline CpG_3 & $0.67 \pm 0.29$ & $2.0 \pm 0.82$ & $0.62 \pm 0.25$ & $1.38 \pm 0.75$ & $0.88 \pm 0.75$ \\
\hline $\mathrm{CpG}_{-4}$ & $11.0 \pm 2.04$ & $8.38 \pm 4.09^{*}$ & $7.75 \pm 2.22$ & $8.0 \pm 1.91$ & $7.25 \pm 1.85$ \\
\hline CpG_5 & $19.0 \pm 3.19$ & $13.2 \pm 2.10^{*}$ & $15.8 \pm 2.90$ & $16.6 \pm 1.11$ & $15.0 \pm 2.16$ \\
\hline \multicolumn{6}{|l|}{ SERPINE1 } \\
\hline CpG_1 & $2.62 \pm 0.63$ & $22.0 \pm 13.7^{* *}$ & $10.8 \pm 14.2$ & $17.0 \pm 16.1^{*}$ & $56.2 \pm 5.14$ \\
\hline CpG_2 & $33.5 \pm 3.39$ & $63.0 \pm 12.5^{* * *}$ & $50.8 \pm 7.09^{* *}$ & $65.0 \pm 5.40^{* * *}$ & $45.4 \pm 8.68^{*}$ \\
\hline CpG_3.4 & $38.5 \pm 9.81$ & $91.1 \pm 8.23^{* *}$ & $79.5 \pm 7.99^{* * *}$ & $85.2 \pm 10.9^{* * *}$ & $62.6 \pm 24.0^{*}$ \\
\hline CpG_6 & $100 \pm 0.00$ & $89.4 \pm 4.09$ & $90.9 \pm 11.3$ & $87.1 \pm 10.2$ & $96.6 \pm 5.49$ \\
\hline CpG_7 & $2.88 \pm 1.60$ & $32.1 \pm 15.4^{* *}$ & $13.9 \pm 7.97^{*}$ & $28.2 \pm 23.1^{*}$ & $15.8 \pm 15.8^{*}$ \\
\hline CpG_8 & $95.1 \pm 2.62$ & $91.5 \pm 6.77$ & $92.6 \pm 6.74$ & $93.1 \pm 2.66$ & $94.9 \pm 2.06$ \\
\hline CpG_9 & $33.5 \pm 3.39$ & $63.0 \pm 12.5^{* *}$ & $50.8 \pm 7.09^{* *}$ & $65.0 \pm 5.40^{* * *}$ & $45.4 \pm 8.68$ \\
\hline CpG_10 & $97.2 \pm 2.59$ & $95.0 \pm 3.03$ & $96.6 \pm 1.60$ & $88.5 \pm 19.7$ & $96.9 \pm 2.46$ \\
\hline CpG_11 & $97.5 \pm 2.91$ & $98.2 \pm 2.36$ & $97.1 \pm 2.69$ & $98.1 \pm 0.75$ & $98.5 \pm 2.68$ \\
\hline CpG_12 & $94.0 \pm 7.22$ & $95.0 \pm 2.42$ & $94.2 . \pm 5.52$ & $96.0 \pm 3.58$ & $92.4 \pm 6.26$ \\
\hline
\end{tabular}

1 (EGR-1), and activator protein 1 (AP-1) [28]. Available data in such research reveals that the binding levels of the $\mathrm{NF}-\kappa \mathrm{B}$ transcription factor to the IL1B promoter were similar to the TNF promoter and to macrophages without the methyl donors' supplementation. These results suggest that DNA methylation does not directly affect the binding affinity of NF- $\kappa$ B to IL1B. Similarly, Feng et al. [17] reported that folic acid decreased TNF- $\alpha$ and IL- $1 \beta$ production by inhibiting the NF- $\kappa \mathrm{B}$ pathway without modifying NF- $\kappa \mathrm{B}$ binding affinity. Taking into account these results, other molecular mechanisms could be affected by the tested molecules. For example, the bioinformatic analysis of the selected sequence of IL1B identified a putative PU.1 (Spi-1) transcription factor binding site, which could be involved in the regulation of the expression of this gene. PU.1 binds to GC-rich regions of genes to activate transcription, hence DNA methylation might impair the binding of PU.1 to the analyzed sequence and downregulate gene transcription. Interestingly, the PU.1 transcription factor is involved in macrophage differentiation and also in the transcriptional control of genes in mature macrophages $[29,30]$.

THP-1 is a human monocytic cell line derived from peripheral blood, which has been widely used to investigate the inflammatory response due to its ability to differentiate into macrophage-like cells. A known limitation of the use of cell lines in research is the difference to the natural environment, however previous studies have found that LPS mimics the inflammatory environment when added to THP-1 [31]. Despite this, direct extrapolation to human disease is not possible because more proinflammatory molecules and more than one cell type are involved in the response. Nevertheless, results of this investigation suggest a direct 

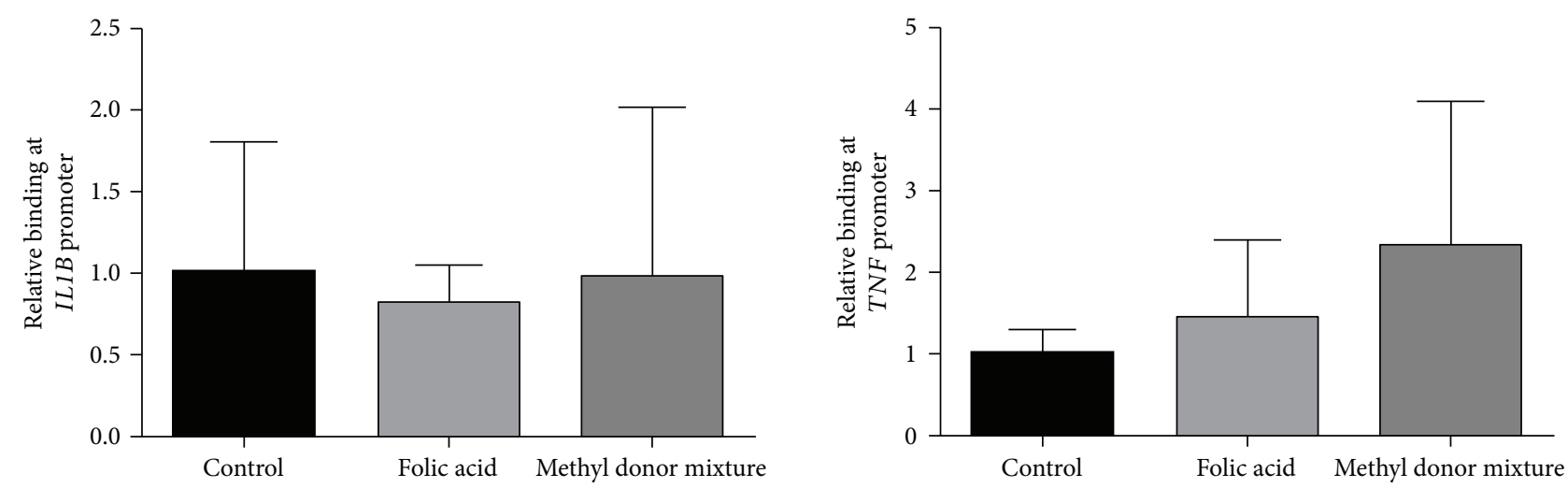

FIGURE 3: Relative binding of NF- $\kappa$ B to IL1B and TNF promoters in THP-1 cells differentiated with TPA and activated with LPS (control group) and the same cells treated with folic acid and the methyl donor mixture before differentiation. Results are expressed as means \pm SD $(n=8)$. Differences between groups were tested by one-way ANOVA.

effect of methyl donors in the methylation of proinflammatory genes in LPS-activated THP-1 cells and the reduction of expression and production of proinflammatory cytokines. In addition, although methyl donor supplementation did not modify TNF promoter methylation, it reduced LPS-induced TNF- $\alpha$ production. However, the binding affinity of NF- $\kappa$ B to proinflammatory genes was unaffected, suggesting a minor role of this protein complex in the transcriptional regulation of these genes in response to folic acid and other methyl donors.

\section{Conclusion}

The findings of this study evidenced that monocyte pretreatment with specific methyl donors, particularly folic acid, reduced the inflammatory response in LPS-activated THP-1 macrophages, which could in part be mediated by increased DNA methylation in some CpG sites of important proinflammatory genes. In addition, folic acid decreased the expression of cytokines and chemokines (i.e., CCL2), suggesting a protective role through the recruitment of monocytes to the inflamed tissue.

\section{Data Availability}

Access to data will be considered by the author upon request.

\section{Conflicts of Interest}

The authors have nothing to declare concerning this issue.

\section{Acknowledgments}

The authors thank the technical assistance of Enrique Buso (UCIM, University of Valencia) for the MassAR$\mathrm{RAY}^{\circledR}$ measurements. The authors are also grateful to Catherine Graham (St Mary's University, UK) for English proofreading. The authors acknowledge the financial support of MINECO (Nutrigenio Project reference AGL201345554-R) and Spanish Centro de Investigación Biomédica en Red de la Fisiopatología de la Obesidad y Nutrición
(CIBERobn). Mirian Samblas holds a FPI grant from the MECD (BES-2014-068409).

\section{Supplementary Materials}

Supplementary Table 1: primer sequences used for MassARRAY EpiTYPER assay. Supplementary Figure 1: genomic localization and nucleotide sequences of $\mathrm{CpG}$ sites covered by the MassARRAY EpiTYPER probes for the study of DNA methylation levels of IL1B, TNF, SERPINE1, and IL18 genes. The number on the left of each sequence represents the positions with respect to the start of the transcription or TSS. Nucleotides in the box are the sequences selected for each gene. CpGs underlined and highlighted in bold are those that were quantified by MassARRAY EpiTYPER. Transcription start site (TSS). Coding DNA sequence (CDS). Supplementary Figure 2: relative cell viability (measured by MTT assay) after THP-1 monocyte incubation with folic acid $(11.3 \mu \mathrm{M})$, choline $(105 \mu \mathrm{M})$, vitamin $B_{12}(18.5 \mathrm{nM})$, and methyl donor mixture. Data are shown as the means \pm SD $(n=6)$. Differences between groups were analyzed by oneway ANOVA. (Supplementary Materials)

\section{References}

[1] G. L. Larsen and P. M. Henson, "Mediators of inflammation," Annual Review of Immunology, vol. 1, no. 1, pp. 335-359, 1983.

[2] D. L. Laskin and K. J. Pendino, "Macrophages and inflammatory mediators in tissue injury," Annual Review of Pharmacology and Toxicology, vol. 35, no. 1, pp. 655-677, 1995.

[3] C. Shi and E. G. Pamer, "Monocyte recruitment during infection and inflammation," Nature Reviews Immunology, vol. 11, no. 11, pp. 762-774, 2011.

[4] O. Fogel, C. Richard-Miceli, and J. Tost, "Epigenetic changes in chronic inflammatory diseases," in Advances in Protein Chemistry and Structural Biology, pp. 139-189, Elsevier, 2017.

[5] J. L. Marques-Rocha, M. Samblas, F. I. Milagro, J. Bressan, J. A. Martínez, and A. Marti, "Noncoding RNAs, cytokines, and inflammation-related diseases," The FASEB Journal, vol. 29, no. 9, pp. 3595-3611, 2015. 
[6] T. C. Simon and M. A. Jeffries, "The epigenomic landscape in osteoarthritis," Current Rheumatology Reports, vol. 19, no. 6, p. 30, 2017.

[7] K. R. Shorter, M. R. Felder, and P. B. Vrana, "Consequences of dietary methyl donor supplements: is more always better?," Progress in Biophysics and Molecular Biology, vol. 118, no. 12, pp. 14-20, 2015.

[8] N. Zhang, "Epigenetic modulation of DNA methylation by nutrition and its mechanisms in animals," Animal Nutrition, vol. 1, no. 3, pp. 144-151, 2015.

[9] A. Cianciulli, R. Salvatore, C. Porro, T. Trotta, and M. A. Panaro, "Folic acid is able to polarize the inflammatory response in LPS activated microglia by regulating multiple signaling pathways," Mediators of Inflammation, vol. 2016, Article ID 5240127, 10 pages, 2016.

[10] Z. Li, R. M. Gueant-Rodriguez, D. Quilliot et al., "Folate and vitamin $B_{12}$ status is associated with insulin resistance and metabolic syndrome in morbid obesity," Clinical Nutrition, 2017.

[11] N. Al-Daghri, S. Rahman, S. Sabico et al., "Association of vitamin $\mathrm{B}_{12}$ with pro-inflammatory cytokines and biochemical markers related to cardiometabolic risk in Saudi subjects," Nutrients, vol. 8, no. 9, article 460, 2016.

[12] A. L. Guerrerio, R. M. Colvin, A. K. Schwartz et al., "Choline intake in a large cohort of patients with nonalcoholic fatty liver disease," The American Journal of Clinical Nutrition, vol. 95, no. 4, pp. 892-900, 2012.

[13] A. L. Buchman, M. D. Dubin, A. A. Moukarzel et al., "Choline deficiency: a cause of hepatic steatosis during parenteral nutrition that can be reversed with intravenous choline supplementation," Hepatology, vol. 22, no. 5, pp. 1399-1403, 1995.

[14] P. Cordero, J. Campion, F. I. Milagro, and J. A. Martinez, "Transcriptomic and epigenetic changes in early liver steatosis associated to obesity: effect of dietary methyl donor supplementation," Molecular Genetics and Metabolism, vol. 110, no. 3, pp. 388-395, 2013.

[15] F. I. Milagro, J. Campión, P. Cordero et al., "A dual epigenomic approach for the search of obesity biomarkers: DNA methylation in relation to diet-induced weight loss," The FASEB Journal, vol. 25, no. 4, pp. 1378-1389, 2011.

[16] C. Lee and C. H. Huang, "LASAGNA-Search 2.0: integrated transcription factor binding site search and visualization in a browser," Bioinformatics, vol. 30, no. 13, pp. 1923-1925, 2014.

[17] D. Feng, Y. Zhou, M. Xia, and J. Ma, "Folic acid inhibits lipopolysaccharide-induced inflammatory response in RAW264.7 macrophages by suppressing MAPKs and NF- $\kappa$ b activation," Inflammation Research, vol. 60, no. 9, pp. 817-822, 2011.

[18] Y. Jiang, S. Ma, H. Zhang et al., "FABP4-mediated homocysteine-induced cholesterol accumulation in THP-1 monocyte-derived macrophages and the potential epigenetic mechanism," Molecular Medicine Reports, vol. 14, no. 1, pp. 969-976, 2016.

[19] A. Solini, E. Santini, and E. Ferrannini, "Effect of short-term folic acid supplementation on insulin sensitivity and inflammatory markers in overweight subjects," International Journal of Obesity, vol. 30, no. 8, pp. 1197-1202, 2006.

[20] G. Wang, J. Dai, J. Mao, X. Zeng, X. Yang, and X. Wang, "Folic acid reverses hyper-responsiveness of LPS-induced chemokine secretion from monocytes in patients with hyperhomocysteinemia," Atherosclerosis, vol. 179, no. 2, pp. 395-402, 2005.
[21] A. F. Kolb and L. Petrie, "Folate deficiency enhances the inflammatory response of macrophages," Molecular Immunology, vol. 54, no. 2, pp. 164-172, 2013.

[22] P. C. Calder, R. Albers, J.-M. Antoine et al., "Inflammatory disease processes and interactions with nutrition," The British Journal of Nutrition, vol. 101, no. S1, pp. 1-45, 2009.

[23] O. S. Anderson, K. E. Sant, and D. C. Dolinoy, "Nutrition and epigenetics: an interplay of dietary methyl donors, one-carbon metabolism and DNA methylation," The Journal of Nutritional Biochemistry, vol. 23, no. 8, pp. 853-859, 2012.

[24] L. Liu, Y. Ke, X. Jiang et al., "Lipopolysaccharide activates ERK-PARP-1-RelA pathway and promotes nuclear factor- $\kappa$ b transcription in murine macrophages," Human Immunology, vol. 73, no. 5, pp. 439-447, 2012.

[25] A. I. Ara, M. Xia, K. Ramani, J. M. Mato, and S. C. Lu, "S-Adenosylmethionine inhibits lipopolysaccharide-induced gene expression via modulation of histone methylation," Hepatology, vol. 47, no. 5, pp. 1655-1666, 2008.

[26] H.-B. Li, J. Tong, S. Zhu et al., " $\mathrm{m}^{6} \mathrm{~A}$ mRNA methylation controls $\mathrm{T}$ cell homeostasis by targeting the IL-7/STAT5/ SOCS pathways," Nature, vol. 548, no. 7667, pp. 338-342, 2017.

[27] X. Wang, B. Sun, Q. Jiang et al., "mRNA $\mathrm{m}^{6} \mathrm{~A}$ plays opposite role in regulating UCP2 and PNPLA2 protein expression in adipocytes," International Journal of Obesity, 2018.

[28] R. Kishore, M. R. McMullen, E. Cocuzzi, and L. E. Nagy, "Lipopolysaccharide-mediated signal transduction: stabilization of TNF-alpha mRNA contributes to increased lipopolysaccharide-stimulated TNF-alpha production by Kupffer cells after chronic ethanol feeding," Comparative Hepatology, vol. 3, article S31, Supplement 1, 2004.

[29] S. Ghisletti, I. Barozzi, F. Mietton et al., "Identification and characterization of enhancers controlling the inflammatory gene expression program in macrophages," Immunity, vol. 32, no. 3, pp. 317-328, 2010.

[30] Z. Yuan, M. A. Syed, D. Panchal, D. Rogers, M. Joo, and R. T. Sadikot, "Curcumin mediated epigenetic modulation inhibits TREM-1 expression in response to lipopolysaccharide," The International Journal of Biochemistry \& Cell Biology, vol. 44, no. 11, pp. 2032-2043, 2012.

[31] R. Ahmad, A. Al-Roub, S. Kochumon et al., "The synergy between palmitate and TNF- $\alpha$ for CCL2 production is dependent on the TRIF/IRF3 pathway: implications for metabolic inflammation," The Journal of Immunology, vol. 200, no. 10, pp. 3599-3611, 2018. 


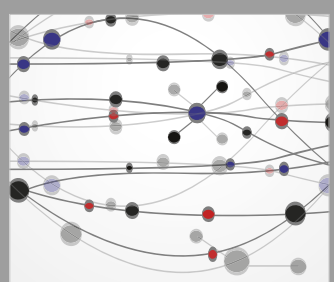

The Scientific World Journal
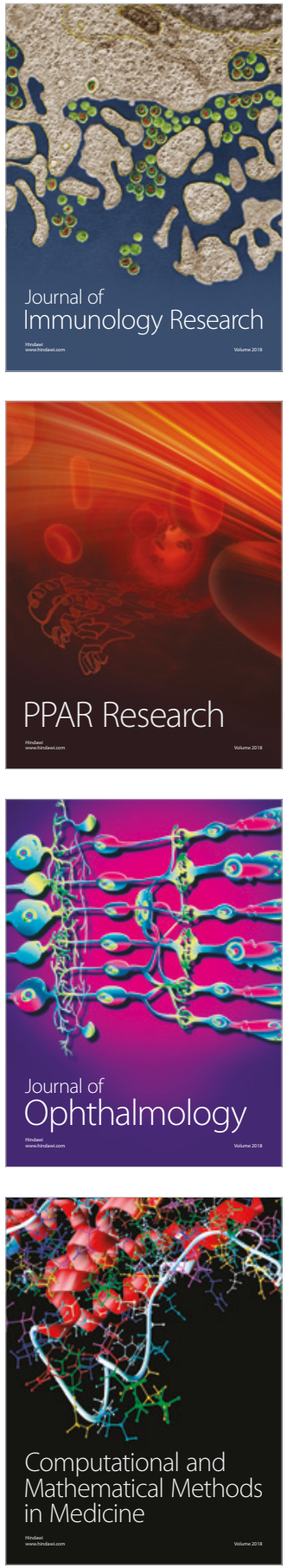

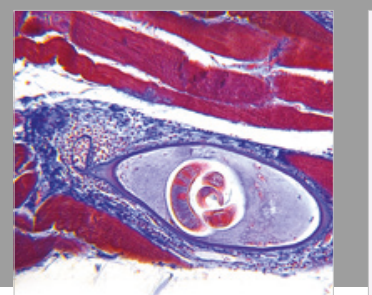

Gastroenterology Research and Practice

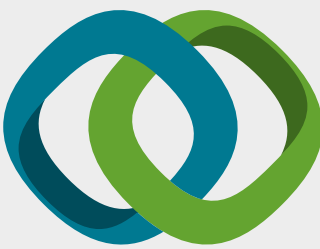

\section{Hindawi}

Submit your manuscripts at

www.hindawi.com
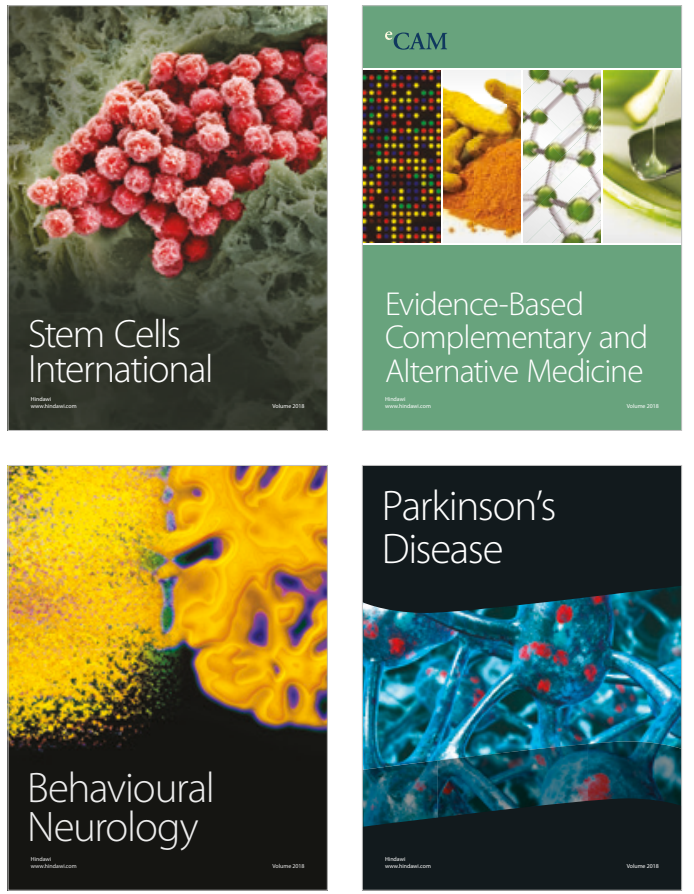

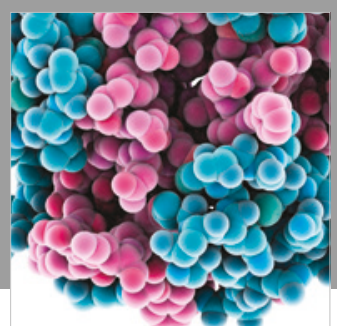

ournal of

Diabetes Research

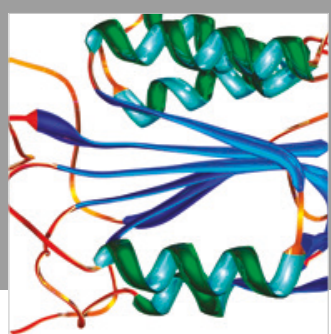

Disease Markers
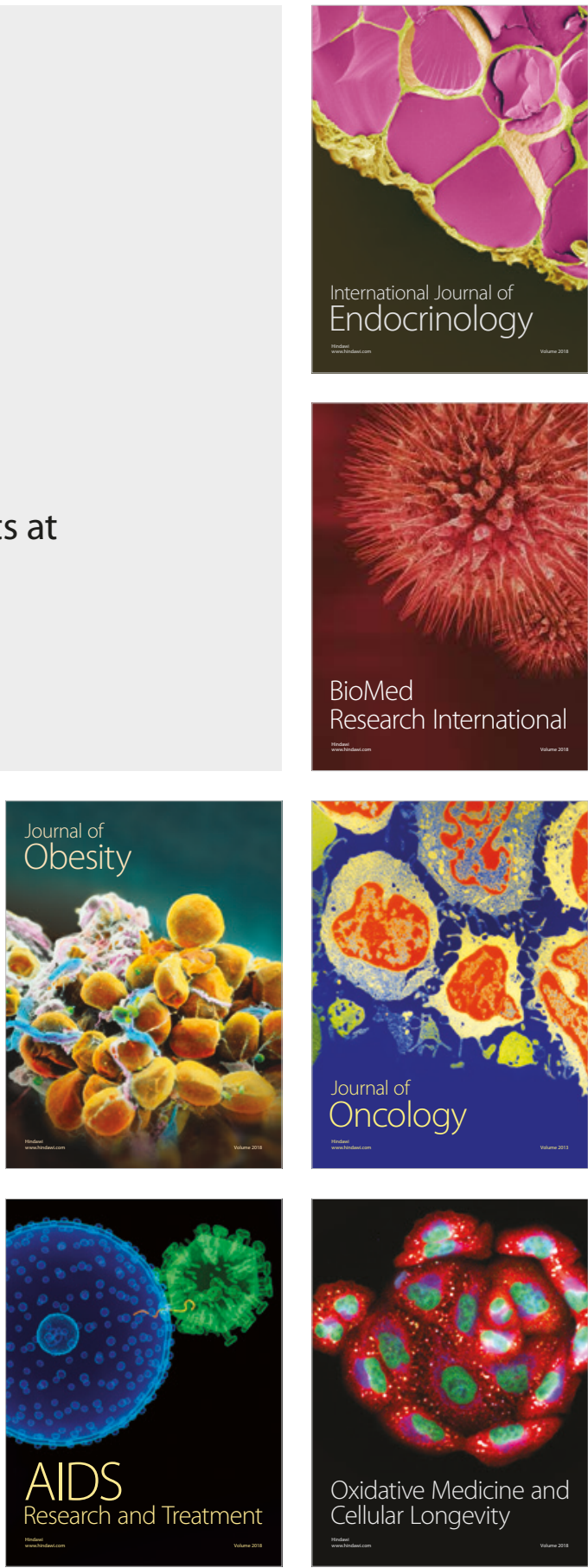